\title{
Predictive Models for the Determination of Pitting Corrosion Versus \\ Inhibitor Concentrations and Temperature \\ for Radioactive Sludge in Carbon Steel Waste Tanks
}

\author{
Jack Leifer \\ University of South Carolina - Aiken \\ Department of Mathematical Sciences and Engineering \\ Aiken, SC 29801 \\ Philip E. Zapp \\ John I. Mickalonis \\ Westinghouse Savannah River Company \\ Savannah River Technology Center \\ Aiken, SC 29808
}

\begin{abstract}
Statistical models have been developed to predict the occurrence of pitting corrosion in carbon steel waste storage tanks exposed to radioactive nuclear waste. The levels of nitrite concentrations necessary to inhibit pitting at various temperatures and nitrate concentrations were experimentally determined via electrochemical polarization and coupon immersion corrosion tests. Models for the pitting behavior were developed based on various statistical analyses of the experimental data. Feed-forward Artificial Neural Network (ANN) models, trained using the Back-Propagation of Error Algorithm, more accurately predicted conditions at which pitting occurred than the logistic regression models developed using the same data.
\end{abstract}

Keywords: pitting corrosion, neural network, logistic regression, data mining

\section{Introduction}

At the U. S. Department of Energy's Savannah River Site near Aiken, SC, high-level radioactive waste (HLW) are stored and processed in large double-walled carbon-steel tanks. The tanks vary in size from 0.75 to 1.3 million gallons. The principal components of the waste are sodium nitrate and the inhibitors sodium hydroxide and nitrite. The waste can induce pitting in the carbon steel if the inhibiting species are depleted. Such pitting is extremely undesirable since perforation of the tank walls would cause a loss of primary containment of the waste and possible contamination of the environment. Both sodium hydroxide and nitrite, when added to and maintained in the HLW in sufficient concentrations prevent, pitting in both the tank walls and the carbon steel cooling coils.

Hydroxide concentrations in excess of $1 \mathrm{M}$ prevent pitting in HLW slurries. When the aqueous phase of the waste is diluted during subsequent processing, the resulting lower hydroxide concentration 
must be supplemented with nitrite to prevent pit initiation. Extensive laboratory experimentation established the necessary minimum nitrite levels for different temperatures. [1,2] The experiments performed were cyclic potentiodynamic polarization (CPP) and coupon immersion, and involved the exposure of ASTM A537 Class 1 carbon steel specimens to non-radioactive simulants of the aqueous phase of dilute HLW. Previous results have shown that testing in simulated waste produced similar results testing in HLW. [1] CPP scans are an accelerated, electrochemical method which allow a rapid determination of the susceptibility of a material to pitting when exposed to a given environment, as well as the minimum levels of inhibitor needed for its prevention. Coupon immersion tests are long-term (120 day) experiments which provide verification of the inhibitor levels determined by the more rapid CPP tests.

In the work described here, waste dilution was characterized by its nitrate concentration, as nitrate is the principal aggressive species. CPP scans were performed at several nitrite concentrations and temperatures with a given waste dilution. The hydroxide concentration was not independently varied. Pitting susceptibility (the presence or absence of pits) was deduced from both the CPP scans and microscopic examination of the CPP specimen. Coupon immersion tests were run at selected nitrite and nitrate concentrations and temperatures similar to those of the CPP scans; the samples were also examined microscopically. A database of pitting behavior for the range of the test variables was developed as a result of this series of experiments. [1]

The experimental data were numerically modeled for predicting the minimum concentration of nitrite required to prevent pitting. Initially, only predictive models incorporating multiple linear regression and logistic regression techniques were tested. [2,3] In order to increase prediction accuracy, artificial neural network (ANN) models were developed and compared with those developed previously. The results showed that ANN models trained with these archival data can be used to determine water chemistries which will limit the probability of pitting to an acceptably low level. As used here, the term archival is meant to indicate a set of data which was not specifically gathered for the purpose of generating a predictive model. Comparison of these models with current operating practices [4] showed that tank waste may be maintained at relaxed conditions without significantly increasing the risk of pitting corrosion. Furthermore, the ANN model was better than the logistic model at predicting the experimental results obtained in the coupon and CPP tests.

\section{Archival Pitting Data}

Zapp and Hobbs [1] obtained a set of 331 experimental measurements linking simulated waste chemistry to pitting corrosion. Both coupon immersion and CPP were used to obtain the data at 180 
discrete combinations of nitrate and nitrite concentrations, and temperature. Nitrate concentrations ranged from $0.025 \mathrm{M}$ to $1.44 \mathrm{M}$; nitrite concentrations from $0.0025 \mathrm{M}$ to $1.20 \mathrm{M}$; and temperatures from $23{ }^{\circ} \mathrm{C}$ to $60{ }^{\circ} \mathrm{C}$. In this series of tests, an experiment for any particular chemistry may have been performed from one to five times. Typically, two experiments were carried out at each reported condition. The complete experimental details have been previously reported. [1]

As is often the case in experimental work, some inconsistencies were found in the results. While most of the reported chemistries had repeatable results (either all pitted or all were passive), a number of chemistries had conflicting results, with some of the samples pitting and some remaining passive. This outcome is not surprising for a number of reasons. Pit formation is a weak function of a number of parameters not taken into consideration here, including surface configuration and condition, solution $\mathrm{pH}$, and the low concentration levels of other chemical species. Conflicting results, therefore, may stem from undocumented differences in experimental conditions. Furthermore, even a water chemistry known to be corrosive is not guaranteed to form a pit - instead it guarantees pit formation to be highly probable. Conflicting results are inherently useful in that they indicate a water chemistry for which the probability of pitting is likely close to neither zero nor one; that is conditions or chemistries that are close to the limit of pitting.

In view of the above issues, the raw experimental data were pruned as follows: (1) All repeated chemistries for which consistent results were obtained were included only once in the pruned data set. Repeated results at the same conditions do not provide any additional information that can improve the numerical models. (2) Chemistries which produced both pitting and passive results were included twice (once for each result). Pruning the data in this manner resulted in a set of 180 chemistries, of which 138 are unique and 42 are conflicting, for a total of 222 points. This set was used to produce a logistic regression model similar to the one reported by Zapp and Edwards [3]. A comparison of logistic regression models produced by the raw and preprocessed data sets is provided in the next section. The pruned data were also used to train and test an Artificial Neural Network model (ANN), which is compared with the logistic models.

\section{Logistic Regression Models}

Zapp and Edwards developed a logistic regression model, using the complete set of 331 experimental data previously [1,3]. A logistic model was initially proposed as a suitable model for predicting pitting probability because the experimental outcome being modeled is binary in nature (i.e., pitting either occurs or does not occur). [3] A complete treatment of the logistic regression method is provided by Hosmer and Lemeshow. [5] 
For the logistic model considered, a functional relationship between the experimental outcome, $y$, (pitting or passive) and the explanatory (test) variables $\left(x_{1}=\log \left[\mathrm{NO}_{3}^{-}\right], x_{2}=\log \left[\mathrm{NO}_{2}^{-}\right]\right.$, and $\mathrm{T}\left[{ }^{\circ} \mathrm{C}\right]$ ) was developed. In this model, the probability $p\left(x_{1}, x_{2}, T\right)$ of pitting at a given set of conditions is expressed as

$$
p\left(x_{1}, x_{2}, T\right)=E\left(y \mid\left(x_{1}, x_{2}, T\right)\right.
$$

where $E\left(y \mid x_{1}, x_{2}, T\right)$ represents the conditional expectation of $\mathrm{y}$ when the values of the explanatory variables $\left(x_{1}, x_{2}, T\right)$ are known. For a set of tests conducted at condition $\left(x_{1}{ }^{*}, x_{2}{ }^{*}, T^{*}\right), p\left(x_{1}{ }^{*}, x_{2}{ }^{*}, T^{*}\right)$ would be equal to the probability that a sample would pit at that condition. Probabilities of $p<0.5$ were taken to correspond to conditions where pitting is not likely to occur; probabilities of $p>0.5$ were assumed to correspond to conditions for which pitting was likely. For natural processes in which the experimental outcome is known to be binary, it has been shown that the plot of $E\left(y \mid x_{1}, x_{2}, T\right)$ often resembles a logistic distribution. [5] For this reason, an attempt was made to fit a logistic model to the pitting susceptibility data. The form of the logistic distribution used to model the probability $p\left(x_{1}, x_{2}, T\right)$ was

$$
p\left(x_{1}, x_{2}, T\right)=1 /\left(1+\exp \left(\mathrm{B}_{0}+\mathrm{B}_{1} x_{1}+\mathrm{B}_{2} x_{2}+\mathrm{B}_{3} T\right)\right)
$$

where $\mathrm{B}_{0}$ is an intercept, $\mathrm{B}_{1}$ is the coefficient of the $\log$ Nitrate term, $\mathrm{B}_{2}$ is the coefficient of the $\log$ Nitrite term, and $\mathrm{B}_{3}$ is the coefficient of temperature. Note that the influence of the explanatory variables in the exponent is linear. Using the statistical software package $\mathrm{SAS}^{1}$ to determine the model coefficients, Zapp and Edwards found the coefficients shown in Table I (Model R), where the coefficient magnitudes indicate the concentrations are more influential than the temperature on the predicted outcome. Of the 238 samples for which the model predicts $0<p<0.5,54$ produced pits, a failure rate of 23 percent. Conversely, of the 93 samples for which $0.5<p<1.0,21$ did not pit, a failure rate of 23 percent. [3] These prediction rates have been summarized in Table II (Model R). As used here, model failure means that for the given conditions, the experimental and predicted outcomes were different. Note that logistic methodology allows the same data to be used to both compute the coefficients found in Equation 2 (i.e., train the model) and to test the model. Such an approach is not used when building Artificial Neural Network models, as separate training and test sets are customarily used. In this case, the inclusion of repeated conditions for which the experimental results are consistent serves to enhance the apparent predictive ability of the logistic model.

The SAS program was used with the pruned data (described in the previous section) to compute a new set of logistic coefficients, shown in Table I (Model P). Of the 160 conditions for which the model

\footnotetext{
${ }^{1}$ SAS is a registered trademark of the SAS Institute, Cary, NC.
} 
predicts $0<p<0.5$ (i.e., no pitting predicted), 55 produced pits, a failure rate of 34 percent. Conversely, of the 62 samples for which $0.5<p<1.0,29$ did not pit, a failure rate of 47 percent.

The logistic model designed using the pruned data set (Model P) is apparently less successful at predicting the experimental results than the model designed using the raw data (Model R). Neither of the results reported in Table II, in fact, provides a true representation of the models' predictive ability. It turns out that the two models are virtually identical! The apparent performance of Model $\mathrm{R}$ is enhanced by the presence of repeated, non-conflicting experimental results. For Model P, however, the maximum performance which can be obtained is limited by the presence of the conflicting experimental results. Therefore, the reported predictive abilities of the models are conjectured to be a strong function of the composition of the data set and its distribution within the experimental domain.

This conjecture is confirmed by using each model to predict probabilities of pitting over a matrix of test conditions which is uniformly distributed over the experimental domain. Such a test set does not allow the performance of any particular model to be evaluated absolutely since there are no experimental results for comparison. However, this test does allow the response of similar models to be compared without being affected by the non-uniform data distribution. This technique can be especially useful for models generated from archival data, such as those used here. As seen from the results shown in Figs. 1-4, both of the models compute similar probabilities of pitting, regardless of the data set used to determine the logistic coefficients. In these figures, probabilities of pitting computed to be greater than 0.75 are taken to be equal to 1.0; probabilities of pitting computed to be less than 0.25 are taken to be equal to 0.0 . The different performance parameters shown in Table II imply that the Models P and R will behave differently over the range of the explanatory variables; yet, Figures 1-4 show virtually identical behavior. This leads to the conclusion that model performance cannot be quantified by only testing against a known set of experimental data because it is in part a function of the data distribution in the test set. This will be true for logistic models, as well as for any numerical model evaluated using experimental data sets, including the ANN models discussed in the next section.

\section{Artificial Neural Network Approach}

Other methods besides logistic regression can be used to construct predictive models from experimental pitting data. One such approach uses an ANN, which is a system that constructs and trains multi-variable, predictive models from experimental data. An ANN serves as a black-box transfer function which links a set of input (explanatory) variables to a set of expected outcome variables. First implemented in the 1950s, ANNs underwent a major increase in popularity during the 1980s and currently comprise an extremely active area of research. ANNs have been used to model widely disparate 
processes, including voice and pattern recognition, chemical structure identification, and fault detection. [6]

In the area of corrosion process modeling, ANNs have been successfully applied by a number of researchers. Rosen and Silverman used an ANN (in conjunction with an expert system) to predict the occurrence of crevice, pitting, and general corrosion from polarization scan parameters. [7] They found that while slightly different ANN implementations produced variations in the corrosion predictions, they were all in general agreement with experimental results. Urquidi-Macdonald and Macdonald compared the predictions of an ANN to predictions obtained using a deterministic model, a statistical Weibull distribution model, and experimental test data. [8] Relatively good agreement was obtained between the number of pits predicted using the ANN and the number of pits experimentally measured as a function of applied potential. They reported that the ANN provides the best agreement with experimental data from among the three models at conditions which produce a high numbers of pits.

\section{ANN: Implementation and Testing}

The topology of the ANN used for this study was a four-layer feed-forward network (Fig. 5) consisting of two hidden layers of processing elements (nodes), an input layer, and an output layer. A sigmoidal transfer function was used in the first three layers; a linear transfer function was chosen for the output layer. The well-known Back-propagation Algorithm was used to train the ANNs developed in this study. A thorough treatment of this type of ANN can be found in Zupan and Gasteiger [6], Haykin [9] and Fausett [10], among others. A commercial Neural Network package, NeuralWorks Professional II Plus $^{2}$ (NWPro) was the development tool used to construct and train the nets developed from the HLW pitting susceptibility data. [11] This program was chosen based on the positive results that previous investigators have had with this package. A multi-layered back-propagation perceptron is one of the many established routines included in this package. The topology of the net (number of nodes and layers), nodal transfer functions, and learning rules, however, are user-specified. The learning scheme was designed to slow learning after $10^{5}$ training cycles. Early in the study, model convergence (minimization of prediction error) did not occur within this number of cycles. As a result, a constant learning rate was used for the scheme adopted. The ANN models described here were trained for about 150000 cycles.

The most widely recognized means of determining the effectiveness of predictive capabilities for ANNs is to divide the experimental data into two parts: a training set and a testing set. By testing the ANN at conditions within the experimental range for which it was not specifically trained, the ability of the ANN to generalize throughout the training range can be verified. Due to the small size of the pruned 
data set $\mathrm{P}$ (222 points), however, the expectation was unreasonable for consistent results to be obtained by dividing the data into individual testing and training sets. Division of the data into training, testing, and (often) verification sets works best when the number of test points ranges from the thousands into the millions and when the experimental data are uniformly distributed throughout the test set. This is clearly not the case here, and the predicted performance of the ANN would be highly dependent upon the distribution of the data in the test set (as was the case for the logistic models). Furthermore, a direct quantitative comparison between the logistic and ANN models would not be possible due to the different compositions of the test set, since the set used to test the ANN would contain less than half the number of points found in Set $\mathrm{P}$.

In view of these problems, an accepted (yet tedious) method, known as "Leave-One-Out," was used for ANN model training and testing. [12] Two hundred twenty-two unique training and testing sets were created from the pruned data (set P). Training set 1 contained all the data in $\mathrm{P}$ except for the first point; test set 1 contained only that first point. In general, the $i^{\text {th }}$ training set contained every point in P except for the $i^{\text {th }}$ point; the corresponding $i^{\text {th }}$ test set contained only the $i^{\text {th }}$ point omitted from the training set. Two hundred twenty-two unique models were thus created and verified from each of the unique training and test sets.

As for the topology of the ANNs themselves, each consisted of 3 input nodes, one output node, and two hidden layers of 5 and 3 nodes each (Figure 5). From this figure, the number of weights can be computed by counting the number of links between the layers, i.e., $(3 X 5)+(5 X 3)+(3 X 1)=33$, a little more than an order of magnitude less than the number of training data (221) present in each set. The ratio of weights to training data is approximately within the "rule-of-thumb" guidelines stated by Fausett [10], Haykin [9], and Dowla and Rogers [12].

Of the 167 conditions for which the ANN predicts no pitting $(0<p<0.5), 52$ produced pits, a failure rate of $31 \%$. Conversely, of the 55 samples for which the ANN predicted pitting $(0.5<p<1.0), 19$ did not pit, a failure rate of $31 \%$. These results are summarized in Table II (Model A).

A comparison of the failure rates of Model A (ANN) and Model P (logistic) results shows that in this case, the ANN model is better able than the logistic model to predict the experimental results. These results are independent of the data distribution, because the same data (set $\mathrm{P}$ ) was used to train and evaluate both the ANN and the logistic models. Although ANN models are believed generally to be better than logistic models for predicting experimental data of this sort, this hypothesis can only be demonstrated by repeating the computer simulations with similar data sets.

\footnotetext{
${ }^{2}$ NeuralWorks Professional II plus is a registered trademark of NeuralWare, Inc., Pittsburgh, PA
} 
For completeness, the ANN model was used to predict pitting probabilities for the uniform matrix of test conditions described previously. The results of this test are shown in Figs. 6-9. As in the plots shown for the two logistic models, probabilities less than 0.25 and greater than 0.75 are shown in the figures as being equal to 0.0 and 1.0, respectively. Although the models are designed using vastly different algorithms, both predict similar trends in pitting probability as a function of water chemistry throughout most of the experimental range although at the higher nitrate concentrations, some divergence in probability occurred.

\section{Discussion}

All of the water chemistries evaluated in this study are considered to promote pitting in this system according to current SRS operating practices [4]. Clearly, based on the results shown in Figs. 1-4 and 6-9, there are chemistries within the experimental domain for which the pitting probability is predicted by both the ANN and logistic models to be at or near zero. Therefore, the predictive models could be used in place of the current technical standards to evaluate waste tank conditions for safe operation. Even if a safety factor is implemented (to increase confidence levels [3]), the region for which the tanks could be operated safely may be enlarged. The range of water chemistries regarded as "safe" would be broadened and; in turn, reduce the amount of required inhibitor, resulting in significant cost savings.

The ultimate disposition of the treated waste is vitrification in the Defense Waste Processing Facility (DWPF) located at SRS. Vitrification is a process in which liquid waste is bonded into a glasslike substance and poured into canisters. Waste stored in this form is 10,000 times more durable and stable than other competing storage methods. [13] Maintaining low levels of nitrite is critical to the vitrification process, since nitrite can cause processing difficulties during the melt and pouring phases, as well as a reduction in quality of the glass itself.

Another advantage of replacing the current Technical Standards with predictive models relates to the models themselves. Since they are constructed and tested using experimental data, the models could be continually updated and improved, as more experimental or operational data became available. Experimental data would be generated through laboratory testing using simulated sludges, and operational data would be obtained by corrosion monitoring. Conducting tests on artificial sludge would be advantageous in that a broader and more uniformly distributed range of chemistries would be studied. Incorporation of these data into the predictive models increases the quality of their predictive capability. 


\section{Conclusions}

The application of new statistical methods to the prediction of pitting was evaluated by developing models using archival experimental pitting data. Test parameters included temperature and concentrations of nitrate (corrosive) and nitrite (inhibitive). The archival data set was modified to eliminate duplicate test results. The ANN-based models were found to provide more accurate predictions than those based upon the logistic regression technique. The prediction rates shown for models $\mathrm{P}$ (logistic) and A (ANN) can only be compared relatively, since the contents of the test data set greatly influence the reported accuracy rates (Table II).

Both models predicted waste chemistries for which no pitting will take place which are within a region outside that allowed by the current Technical Standards used to operate the waste tanks. These predictive models, therefore, could be used to relax the current restrictions, which would provide cost savings. Confidence in the models' predictive abilities should be enhanced by completing a new series of pitting tests, to supplement the data currently available. These tests should be conducted at uniformly distributed points throughout the domain of interest, which would improve the overall general quality and allow a more quantitative evaluation of the models' predictive ability.

\section{Acknowledgements}

This research was funded by the South Carolina University Research and Education Foundation (SCUREF) Task Order 189 through the U.S. Department of Energy contract DE-AC09-89SR18035.

\section{$\underline{\text { References }}$}

1. P. E. Zapp and D. T. Hobbs, in CORROSION/92, paper no. 98, NACE International, Houston, TX (1992).

2. J. W. Congdon, Mat Perf., 21, (1988):p. 34.

3. P. E. Zapp and T. B. Edwards, in CORROSION/94, paper no. 116, NACE International, Houston, TX (1994).

4. "Process Requirements, 241-82H Control Room, Savannah River Site," Report No. WSRC-IM-9163, Westinghouse Savannah River Company, Aiken, SC, June 1996. 
5. D. W. Hosmer, Jr., and S. Lemeshow, Applied Linear Regression, (New York: John Wiley and Sons, 1989).

6. J. Zupan and J. Gasteiger, Neural Networks for Chemists, (New York: VCH Publishers, 1993).

7. E. M. Rosen and D. C. Silverman, Corrosion, 48, 9 (1992): p. 734.

8. M. Urquidi-Macdonald and D. D. Macdonald, J Res. Natl. Inst. Stand Technol., 99, 4 (1994): p. 495.

9. S. Haykin, Neural Networks: A Comprehensive Foundation, (New York: IEEE, 1994).

10. L. Fausett, Fundamentals of Neural Networks, (Saddle River, NJ: Prentice Hall, 1994).

11. NeuralWorks Professional II/Plus, Reference Manual (Pittsburgh, PA: NeuralWare, Inc., 1995).

12. F. U. Dowla and L. L. Rogers, Solving Problems in Environmental Engineering and Geosciences with Artificial Neural Networks, (Cambridge, MA: The MIT Press, 1995).

13. J. T. Carter, K. J. Rueter, J. W. Ray and O. Hodoh, "Defense Waste Processing Facility Radioactive Operation - Part II Glass Making,” WSRC-MS-96-0798, Westinghouse Savannah River Company, December 1996. 
Captions: Leifer, Mickalonis and Zapp, "Predictive Models for the Determination of Pitting Corrosion versus Inhibitor Concentrations and Temperature for Radioactive Sludge in Carbon Steel Tanks"

Figure 1: Comparison of pitting probabilities as predicted by two logistic models: $\left[\mathrm{NO}_{3}\right]=0.032$ ppm. Prediction of pitting shown as a function of nitrite concentration (as indicated) and temperature at $5{ }^{\circ} \mathrm{C}$ intervals.

Figure 2: Comparison of pitting probabilities as predicted by two logistic models: $\left[\mathrm{NO}_{3}\right]=0.10$ $\mathrm{ppm}$. Prediction of pitting shown as a function of nitrite concentration (as indicated) and temperature at $5{ }^{\circ} \mathrm{C}$ intervals.

Figure 3: Comparison of pitting probabilities as predicted by two logistic models: $\left[\mathrm{NO}_{3}\right]=0.32$ ppm. Prediction of pitting shown as a function of nitrite concentration (as indicated) and temperature at $5{ }^{\circ} \mathrm{C}$ intervals.

Figure 4: Comparison of pitting probabilities as predicted by two logistic models: $\left[\mathrm{NO}_{3}\right]=1.00$ ppm. Prediction of pitting shown as a function of nitrite concentration (as indicated) and temperature at $5^{\circ} \mathrm{C}$ intervals.

Figure 5: Four-layer feed forward network. Nodes are represented by open circles, links/weights by the lines connecting the layers of nodes.

Figure 6: Comparison of pitting probabilities as predicted by ANN and logistic models: $\left[\mathrm{NO}_{3}\right]=$ $0.032 \mathrm{ppm}$. Prediction of pitting shown as a function of nitrite concentration (as indicated) and temperature at $5{ }^{\circ} \mathrm{C}$ intervals.

Figure 7: Comparison of pitting probabilities as predicted by ANN and logistic models: $\left[\mathrm{NO}_{3}\right]=$ $0.10 \mathrm{ppm}$. Prediction of pitting shown as a function of nitrite concentration (as indicated) and temperature at $5{ }^{\circ} \mathrm{C}$ intervals.

Figure 8: Comparison of pitting probabilities as predicted by ANN and logistic models: $\left[\mathrm{NO}_{3}\right]=$ $0.32 \mathrm{ppm}$. Prediction of pitting shown as a function of nitrite concentration (as indicated) and temperature at $5{ }^{\circ} \mathrm{C}$ intervals.

Figure 9: Comparison of pitting probabilities as predicted by ANN and logistic models: $\left[\mathrm{NO}_{3}\right]=$ $1.00 \mathrm{ppm}$. Prediction of pitting shown as a function of nitrite concentration (as indicated) and temperature at $5{ }^{\circ} \mathrm{C}$ intervals.

Table I: Coefficients for Logistic Models (Equation 2)

Table II: Comparison of Model Performance 
WSRC-MS-98-00653

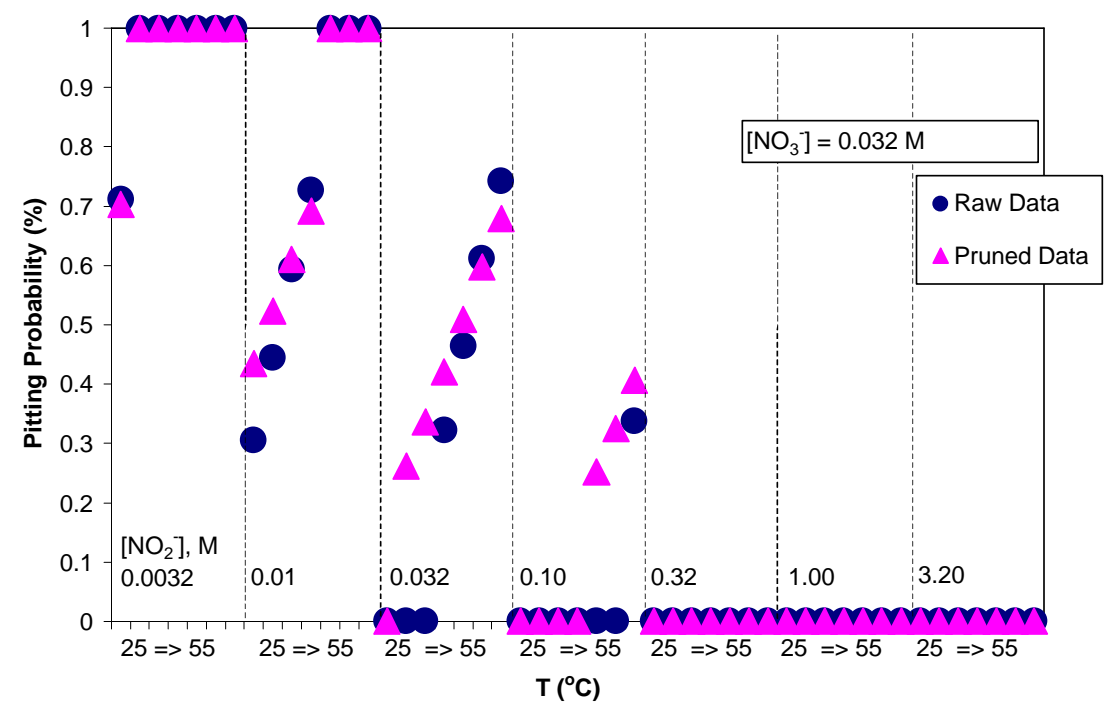

Figure 1: Comparison of pitting probabilities as predicted by two logistic models: $\left[\mathrm{NO}_{3}\right]=0.032$ $\mathrm{ppm}$. Prediction of pitting shown as a function of nitrite concentration (as indicated) and temperature at $5{ }^{\circ} \mathrm{C}$ intervals.

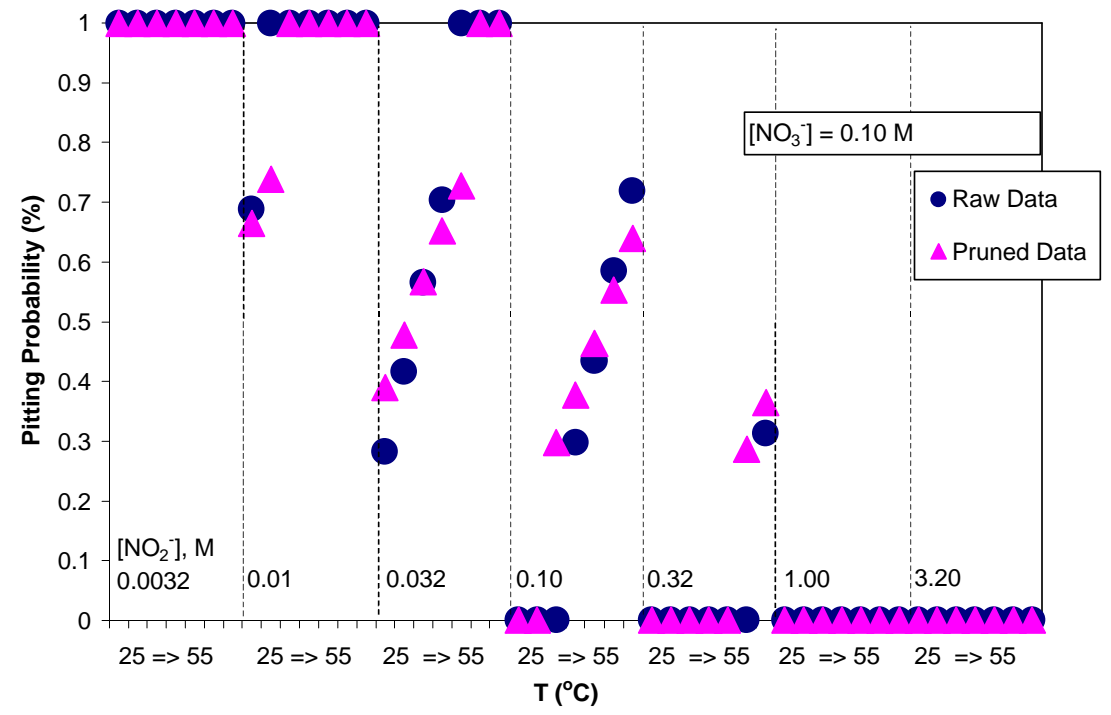

Figure 2: Comparison of pitting probabilities as predicted by two logistic models: $\left[\mathrm{NO}_{3}\right]=0.10$ ppm. Prediction of pitting shown as a function of nitrite concentration (as indicated) and temperature at $5{ }^{\circ} \mathrm{C}$ intervals. 
WSRC-MS-98-00653

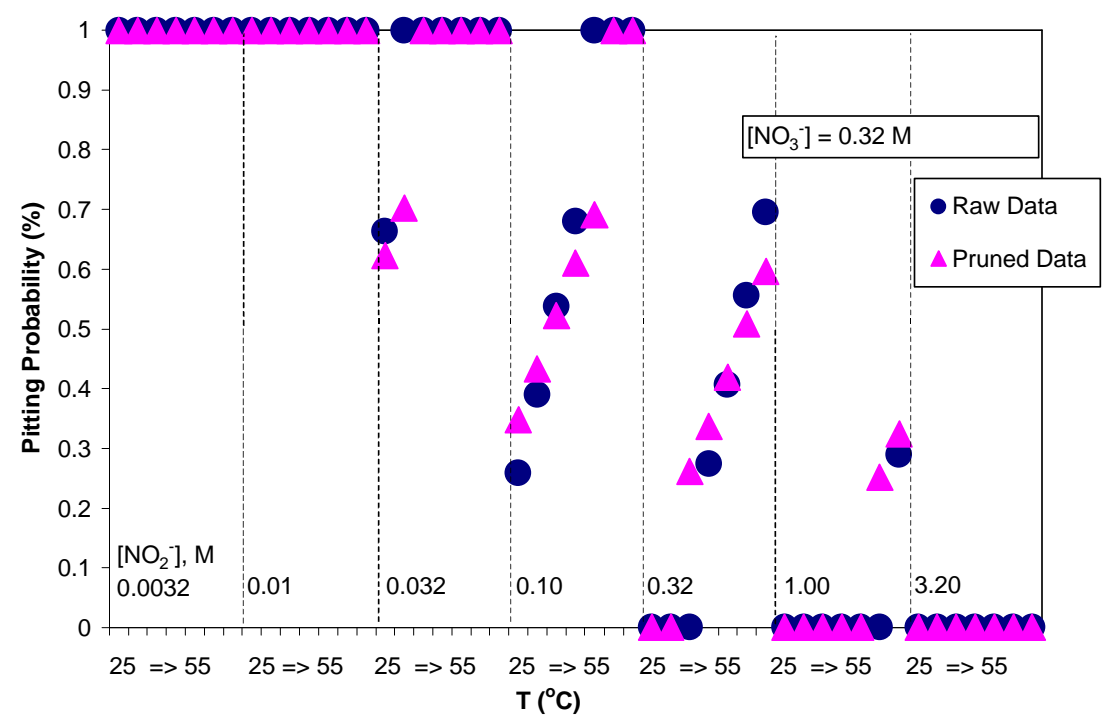

Figure 3: Comparison of pitting probabilities as predicted by two logistic models: $\left[\mathrm{NO}_{3}\right]=0.32$ ppm. Prediction of pitting shown as a function of nitrite concentration (as indicated) and temperature at $5{ }^{\circ} \mathrm{C}$ intervals.

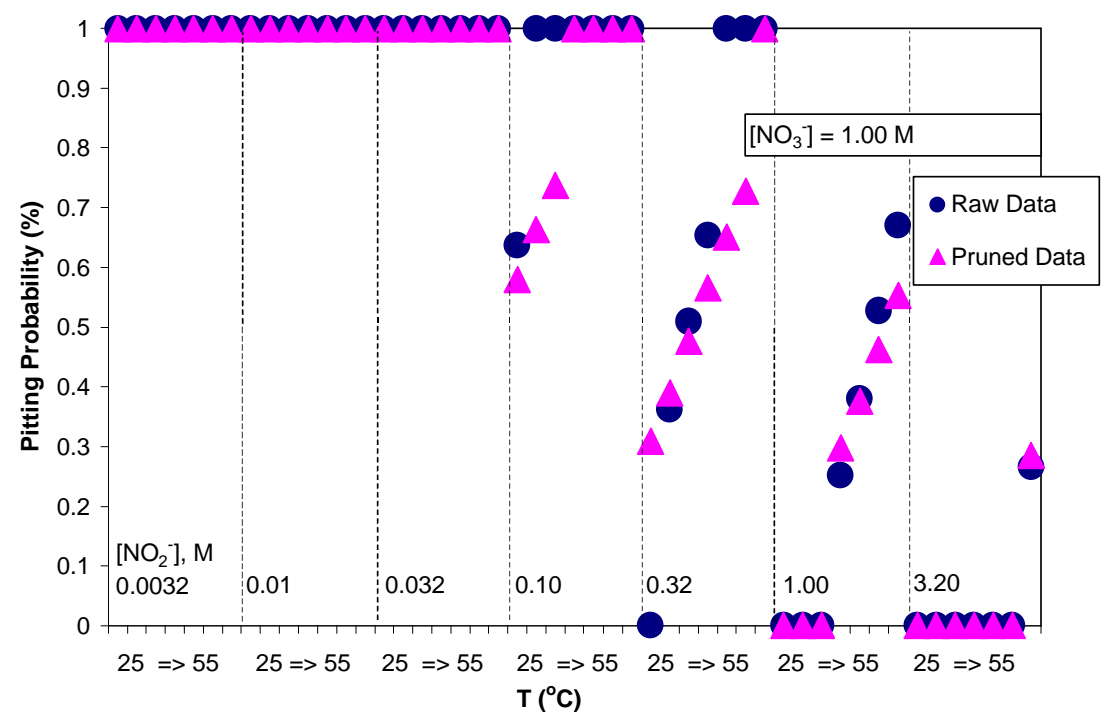

Figure 4: Comparison of pitting probabilities as predicted by two logistic models: $\left[\mathrm{NO}_{3}\right]=1.00$ $\mathrm{ppm}$. Prediction of pitting shown as a function of nitrite concentration (as indicated) and temperature at $5{ }^{\circ} \mathrm{C}$ intervals. 


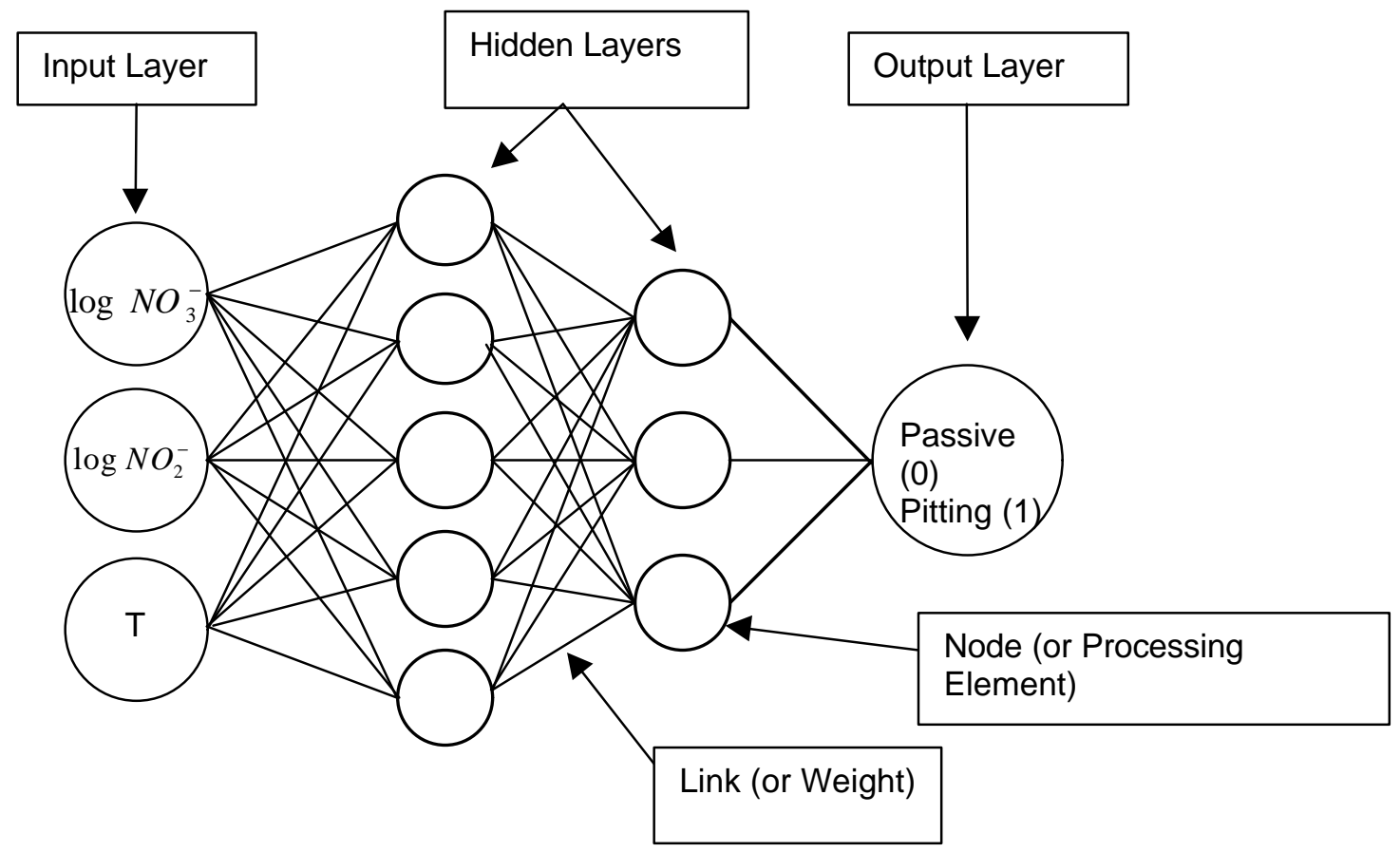

Figure 5: Four-layer feed forward network. Nodes are represented by open circles, links/weights by the lines connecting the layers of nodes. 
WSRC-MS-98-00653

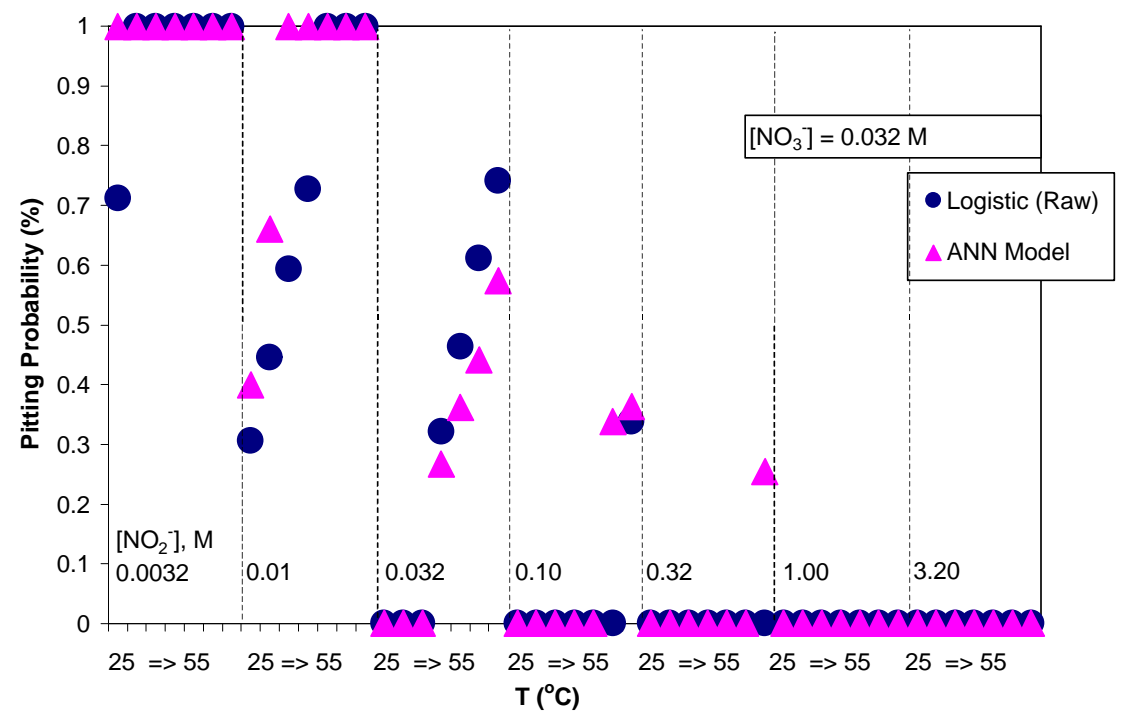

Figure 6: Comparison of pitting probabilities as predicted by ANN and logistic models: $\left[\mathrm{NO}_{3}\right]=$ $0.032 \mathrm{ppm}$. Prediction of pitting shown as a function of nitrite concentration (as indicated) and temperature at $5{ }^{\circ} \mathrm{C}$ intervals.

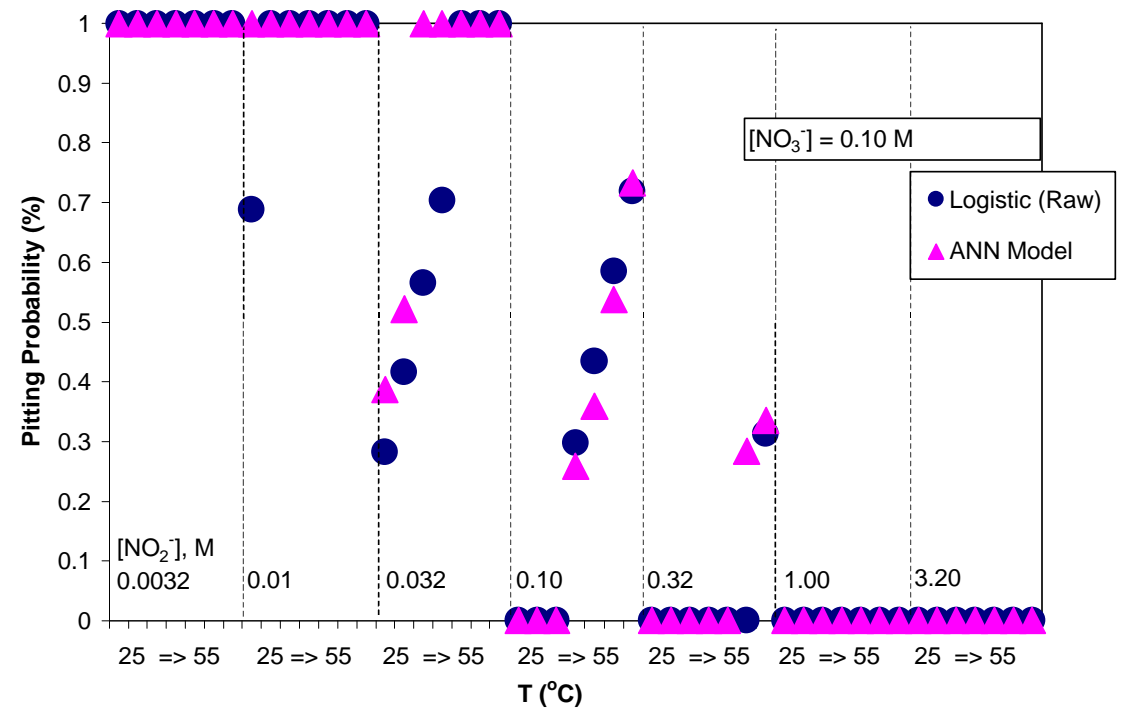

Figure 7: Comparison of pitting probabilities as predicted by ANN and logistic models: $\left[\mathrm{NO}_{3}\right]=$ $0.10 \mathrm{ppm}$. Prediction of pitting shown as a function of nitrite concentration (as indicated) and temperature at $5{ }^{\circ} \mathrm{C}$ intervals. 
WSRC-MS-98-00653

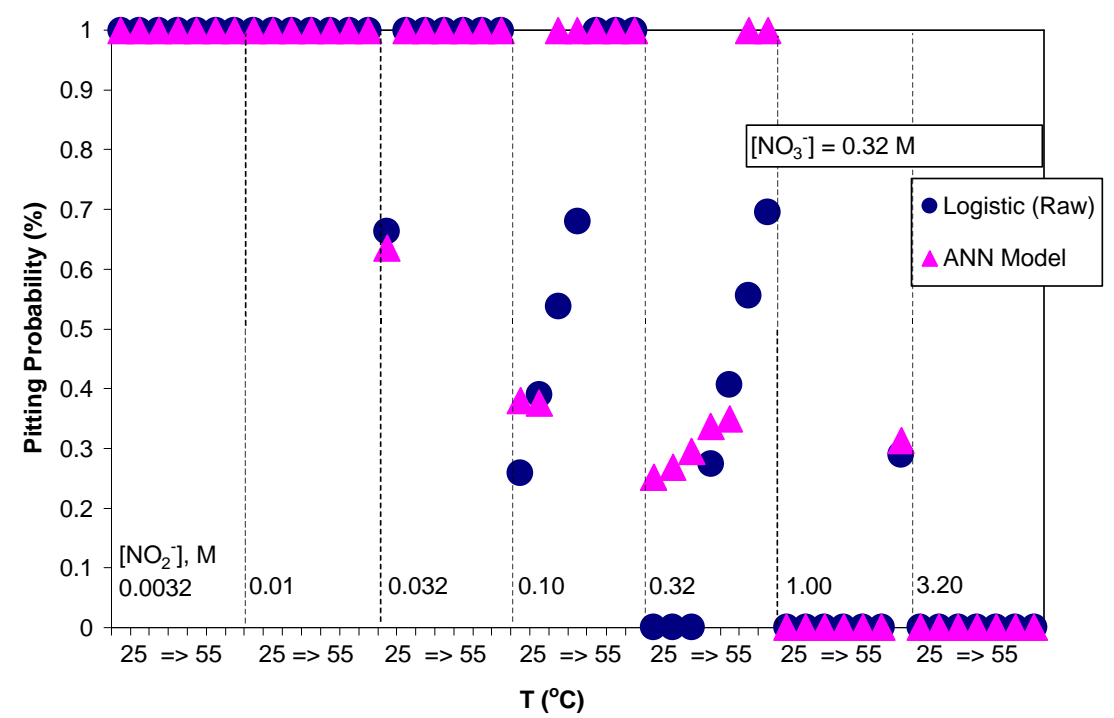

Figure 8: Comparison of pitting probabilities as predicted by ANN and logistic models: $\left[\mathrm{NO}_{3}\right]=$ $0.32 \mathrm{ppm}$. Prediction of pitting shown as a function of nitrite concentration (as indicated) and temperature at $5{ }^{\circ} \mathrm{C}$ intervals.

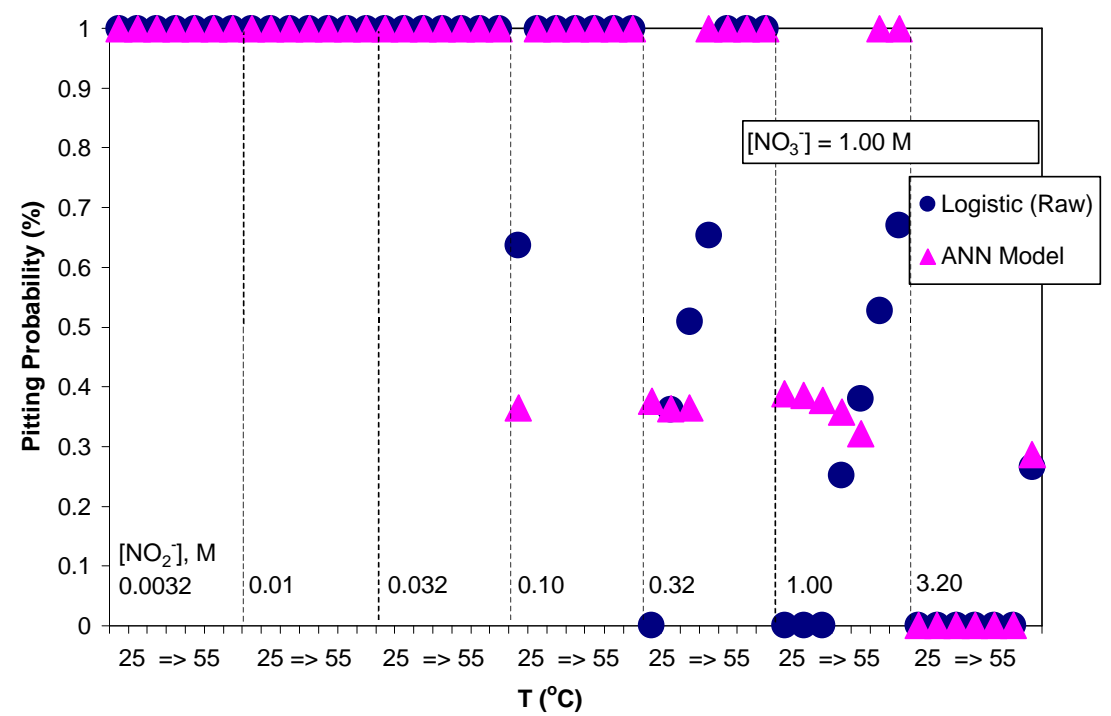

Figure 9: Comparison of pitting probabilities as predicted by ANN and logistic models: $\left[\mathrm{NO}_{3}\right]=$ $1.00 \mathrm{ppm}$. Prediction of pitting shown as a function of nitrite concentration (as indicated) and temperature at $5{ }^{\circ} \mathrm{C}$ intervals. 
Table I: Coefficients for Logistic Models (Equation 2)

\begin{tabular}{|l|l|l|l|l|}
\hline Model & $\mathrm{B}_{0}$ & $\mathrm{~B}_{1}$ & $\mathrm{~B}_{2}$ & $\mathrm{~B}_{3}$ \\
\hline $\mathrm{R}(331$ Points) & 5.89 & -3.22 & 3.45 & -0.12 \\
\hline $\mathrm{P}(222$ Points) & 3.712 & -1.89 & 2.25 & -0.0713 \\
\hline
\end{tabular}

Table II: Model Performance

\begin{tabular}{|l|l|l|}
\hline & $\begin{array}{l}\text { Predicted to Pit/ } \\
\text { Pit Formed (\%) }\end{array}$ & $\begin{array}{l}\text { Predicted Not to Pit// } \\
\text { No Pit Formed (\%) }\end{array}$ \\
\hline $\begin{array}{l}\text { Logistic } \\
\text { Model R }\end{array}$ & 77.4 & 77.3 \\
\hline $\begin{array}{l}\text { Logistic } \\
\text { Model P }\end{array}$ & 53 & 66 \\
\hline $\begin{array}{l}\text { ANN } \\
\text { Model A }\end{array}$ & 69 & 69 \\
\hline
\end{tabular}

